\title{
Avaliação sensorial do guacamole com adição de $\alpha$-tocoferol e ácido ascórbico conservado pelo frio*
}

\author{
Érica Regina Daiuto ${ }^{1}$; Rogério Lopes Vieites², Lídia Raquel de Carvalho³, Juliana Wagner Simon ${ }^{4}$, \\ Viviane Citadini Russo 5
}

\begin{abstract}
RESUMO
A comercialização do abacate processado e sem aditivo químico é difícil, por causa do escurecimento de sua polpa e alterações sensoriais durante o armazenamento. O objetivo desta pesquisa foi verificar a aceitação do guacamole, processado com a adição $\alpha$-tocoferol e ácido áscorbico e conservado pelo frio. Amostras do produto foram acondicionadas em embalagens de polietileno e de polietileno+náilon, com e sem aplicação de vácuo. As amostras embaladas foram submetidas aos tratamentos pelo frio: refrigeração, congelamento lento e congelamento rápido. Avaliações foram realizadas nos dias $0,1,3,5,7$, para o tratamento refrigerado, e 0, 7, 30, 60, 90 dias, para as amostras submetidas aos congelamentos lento e rápido. Para avaliação sensorial, utilizou-se o método de aceitação (escala hedônica estruturada de 9 pontos), com um grupo de 40 provadores não treinados, selecionados ao acaso, que deram notas para aceitação, aparência, cor, textura e sabor. A aceitação do produto e seu sabor tiveram uma correlação de 0,84 , que, para cor e aparência, foi de 0,74. Para as demais variáveis, as correlações foram moderadas. A análise sensorial mostrou que a adição de ácido ascórbico e $\alpha$-tocoferol permite a conservação do produto sob refrigeração em embalagens de polietileno. O produto, conservado até 30 dias, em qualquer embalagem, apresentou médias dos parâmetros de aceitação comparáveis com as obtidas na avaliação do primeiro dia.
\end{abstract}

Palavras-chave: Persea americana Mill, refrigeração, congelamento, processamento, antioxidantes, aceitação.

\section{ABSTRACT}

\section{Sensory analysis of cold-stored guacamole added with $\alpha$-tocopherol and ascorbic acid}

Marketing of processed avocado without chemical additives is difficult. Cold temperature causes browning and sensory changes during storage. The aim of this study was to evaluate the acceptance of cold-stored guacamole added with $\alpha$-tocopherol and ascorbic acid. Guacamole samples were packaged in polyethylene and polyethylene+nylon bags with and without vacuum pump application. Packaged samples were treated by refrigeration, slow and fast freezing. Sensory evaluations were performed on days $0,1,3,5,7$ days after initiation of the refrigeration treatment and days 7, 30, 60, 90 after initiation of slow and fast freezing. Sensory evaluations were carried out using a 9 point hedonic scale with a group of forty randomly selected, untrained panelists to score samples with respect to acceptance, appearance, color, texture and flavor parameters. Correlation between acceptance and flavor was 0.84 and between color and appearance was 0.74 . Moderate correlations were found between the other variables. The sensorial analysis showed that the addition of ascorbic acid and $\alpha$-tocopherol allows preservation of guacamole by refrigeration in polyethylene packaging. The product preserved up to 30 days in any packaging components showed means of acceptance scores close to values of the evaluation at the first day.

Key words: Persea americana Mill, refrigeration, freezing, processing, antioxidant, acceptance.

Recebido para publicação em outubro de 2009 e aprovado em março de 20101

Projeto de pós doutorado com apoio da CAPES, programa PNPD e FAPESP.

${ }^{1}$ Engenheira-Agrônoma, Doutora. Pós doutoranda no Curso de Horticultura da FCA/UNESP, Rua Tulipa, 42, Vila Paraíso, 18607060, Botucatu-SP, Brasil. erdaiuto@uol.com.br; ${ }^{2}$ Engenheiro-Agrônomo, Doutor. Departamento de Gestão e Tecnologia de Alimentos, FCA/UNESP, Fazenda Experimental Lageado, 18610307, CP 237, Botucatu-SP, Brasil. vieites@fca.unesp.br ;

3 Matemática, Doutora. Departamento de Bioestatística IB/UNESP, Distrito de Rubião Júnior 18618-970, Botucatu-SP, Brasil, lidiarc@ibb.unesp.br;

${ }^{4}$ Farmacêutica, Mestre. Fazenda Experimental Lageado, 18610307, CP 237, Botucatu-SP, Brasil. julianasimon@fca.unesp.br;

${ }^{5}$ Engenheira-Agrônoma. Mestranda FCA/UNESP, Fazenda Experimental Lageado, 18610307, CP 237, Botucatu-SP, Brasil. vivianecitadini@hotmail.com 


\section{INTRODUÇÃO}

O abacate (Persea americana Mill.) é um fruto climatérico, com alta taxa respiratória e produção elevada de etileno após a colheita; portanto, altamente perecível sob condições ambientais (Kader, 1992). O controle do amadurecimento é fundamental para o aumento da vida útil após colheita, visando ao mercado interno e à exportação de frutas (Kluge et al., 2002). Já a comercialização do fruto na forma processada é um grande desafio, pois a polpa escurece rapidamente depois de cortada, por presença de enzimas responsáveis pelo escurecimento, principalmente a polifenoloxidase (Bates, 1968; Gómez-Lopez, 2002; Luíz et al., 2007).

Abacates 'Hass' e 'Fuerte', que apresentam diâmetros menores, têm sido cultivados no interior de São Paulo e são exportados, com selo de certificação, conquistando também o mercado interno. $\mathrm{O}$ abacate na forma processada é uma alternativa para aproveitar os frutos de qualidade, porém fora de padrão para comercialização ou exportação, além de se obter um produto de maior valor agregado.

Dentre os produtos processados de abacate, de que se têm relatos de pesquisas na literatura, encontram-se a polpa, a pasta, o purê e o guacamole. O guacamole é um produto composto por polpa do fruto, sal, cebola, limão, pimenta e tomate, sendo elaborado não só de forma artesanal, mas, também, comercializado por algumas empresas norte- americanas.

Vários pesquisadores, em diversos países do mundo, tentam a obtenção de uma polpa estável de abacate, utilizando uma série de métodos de preservação, tais como: pasteurização, secagem, extração de óleo, congelamento, liofilização e, mais recentemente, o tratamento sob alta pressão (Cruess et al. 1951, apud Stephens et al., 1958; Palou et al., 2000; Soliva et al., 2001; Soliva-Fortuny et al., 2004,). Guzmán et al. (2002) avaliaram o uso do aquecimento em micro-ondas, aliado ao uso de cloreto de cobre, na preservação da cor do purê de abacate. Usam-se, também, agentes químicos redutores, sequestrantes e ácidos (Son et al., 2000). Essas substâncias podem ser usadas em processos combinados, como tratamentos sob alta pressão (López-Malo, et al. 1998).

O tratamento térmico, também utilizado, pode alterar a granulosidade do guacamole, tornando sua aparência a de um purê ou pasta. Apesar de esse método ser um tratamento efetivo para inativação da polifenoloxidase, pode resultar no desenvolvimento de gosto amargo e de offflavors no abacate (Ben-Et et al., 1973).

Produtos vegetais com alto teor de óleo, como o abacate, são sensíveis ao processo de oxidação, resultando em rancidez, consequentemente na produção indesejável de flavor e perda da qualidade durante o armazenamento (Gunstone \& Norris, 1982 apud ElezMartinez et al., 2005). Elez-Martinez et al. (2005) demonstraram a possibilidade de controle da rancidez oxidativa de purê de abacate, processado com o uso de $\alpha$-tocoferol e ácido ascórbico.

O ácido ascórbico e o $\alpha$-tocoferol são antioxidantes naturais (Schwenke, 1998), cuja ação pode-se dar, teoricamente, ligando-se competitivamente ao oxigênio, retardando a etapa de iniciação, interrompendo a etapa de propagação, pela destruição ou pela ligação de radicais livres, inibindo os catalisadores ou estabilizando os dihidroxiperóxidos (Reginato D'arce, 2006). Componentes como o $\beta$ caroteno agem inibindo o oxigênio singleto e interagem sinergicamente com a vitamina $\mathrm{E}$, para inibir a peroxidação lipídica (Schwenke, 1998). Já o ácido ascórbico é um antioxidante sinergístico e removedor de oxigênio. Age diretamente com o oxigênio, formando o deidroacético ascórbico e eliminando o suprimento de oxigênio disponível para reações de auto-oxidação (Araújo, 2006). O ácido ascórbico atua como poderoso antioxidante na fração aquosa do abacate, especialmente prevenindo reações de escurecimento (Soliva- Fortuny et al., 2001).

A qualidade sensorial do guacamole, conservado pelo frio, produzido com a variedade (Hass), sem aditivos químicos, foi avaliada por Daiuto et al. (2007). Os resultados mostraram que as amostras acondicionadas em embalagem com barreira contra gases resultam em notas melhores para os parâmetros de aceitação avaliados, sendo que as piores avaliações foram para embalagens de polietileno sob armazenamento refrigerado. Nessa pesquisa, os resultados mostraram ser possível a conservação do produto sob refrigeração.

No Brasil, o abacate processado ou minimamente processado não é comercializado, como em outros países, que chegam a exportar a pasta ou guacamole. Isso se deve a diferenças nos hábitos de consumo. No entanto, alguns restaurantes, principalmente franquias de países estrangeiros, têm procurado pelo produto, no Brasil. A redução do uso de aditivos em alimentos é uma tendência atual, uma vez que, os consumidores procuram por produtos práticos, de boa qualidade e com características idênticas ao produto fresco.

Esta pesquisa visa verificar a aceitação do guacamole produzido com a adição $\alpha$-tocoferol e ácido ascórbico conservado pelo frio.

\section{MATERIAL E MÉTODOS}

\section{Matéria prima}

A elaboração do guacamole foi realizada com frutos de abacate da variedade Hass. Os demais ingredientes, como molho de pimenta, sal, limão, cebola e tomate, foram obtidos em supermercado. O limão utilizado foi o Taiti. 


\section{Processamento}

Os frutos, em estado adequado de maturação, foram selecionados para obtenção do guacamole e o procedimento de preparo foi manual, seguindo as boas práticas de manipulação de alimentos, sendo o processo esquematizado na Figura 1. A polpa foi desintegrada, com uma faca, em pedaços pequenos, sem, no entanto, formar uma pasta. A seguir, foi adicionada a cebola, que foi previamente desintegrada em liquidificador doméstico, apresentando forma de uma pasta, e, o tomate, em quadrados de 1 $\mathrm{cm}$. Os ingredientes secos (sal e aditivos) foram misturados na pimenta ou no limão e homogeinizados, com auxílio de uma colher, junto aos outros ingredientes. Foram adicionados $0,5 \%$ de $\alpha$-tocoferol e $1 \%$ de ácido ascórbico sobre o total da formulação. Na formulação utilizou-se, para a proporção de $500 \mathrm{~g}$ de polpa de abacate, $20 \mathrm{~g}$ de limão, 7,5 g de molho de pimenta, 15 gramas de cebola, 70 $\mathrm{g}$ de tomate e 5,5 $\mathrm{g}$ de sal.

\section{Tratamentos}

Após o preparo, o guacamole foi armazenado em sacos de polietileno e embalagens de náilon+polietileno (barreira contra gases), sendo submetido a condições de refrigeração $(\mathrm{R})$ a aproximadamente $4^{\circ} \mathrm{C}$, congelamento lento (CL) em freezer doméstico $-18^{\circ} \mathrm{C}$, e congelamento rápido (CR), por 30 min a $-18^{\circ} \mathrm{C}$, em aparelho Irinox-refrigerador HCFC 22. Amostras acondicionadas em sacos de polietileno $(\mathrm{P})$, náilon+polietileno $(\mathrm{SV})$ e náilon+polietileno com vácuo $(\mathrm{V})$, sob refrigeração, foram avaliadas no $1^{\circ}, 3^{\circ}$, $5^{\circ}$ e $7^{\circ}$ dias de armazenamento. As amostras congeladas

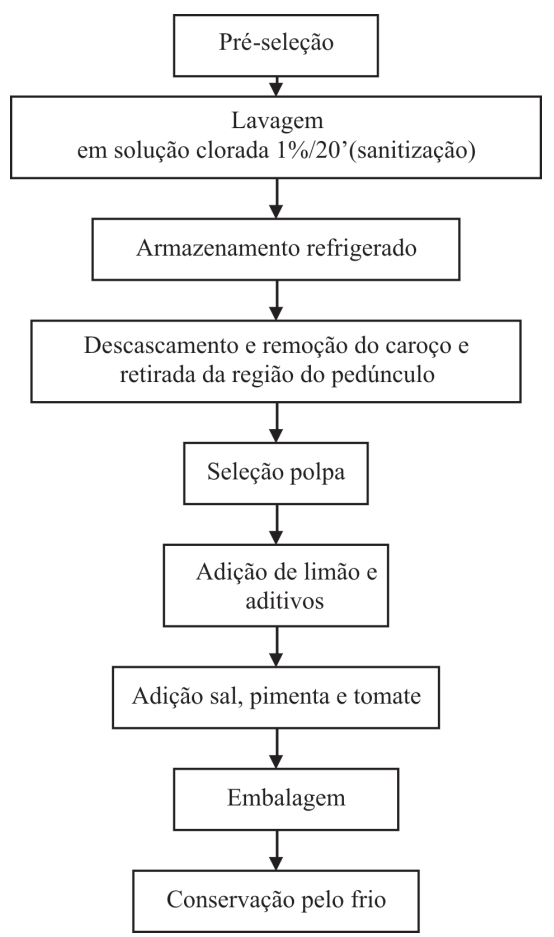

Figura 1 - Fluxograma do processamento do guacamole. foram acondicionadas no mesmo tipo de embalagem e analisadas aos 7, 30, 60 e 90 dias. As amostras submetidas aos congelamentos lento e rápido foram armazenadas em freezer domésticos a $-18^{\circ} \mathrm{C}$. Foi feita uma avaliação no dia de elaboração do produto (T0). As análises foram realizadas até que a qualidade fosse aceitável para o consumidor, levando em consideração aspecto visual e coloração.

\section{Análise Sensorial}

A avaliação sensorial foi realizada, utilizando-se o método de aceitação (Chaves \& Sproesser, 1999), em condições laboratoriais, com um grupo de 40 provadores não treinados, escolhidos ao acaso. O delineamento utilizado foi de blocos ao acaso. Foram aplicadas fichas indicando os parâmetros aparência, cor, textura, sabor e aceitação. Um modelo da ficha é apresentado na Figura 2. Os provadores foram orientados a marcar com um traço vertical na escala de 1 a 9 pontos, o quanto gostaram ou desgostaram do produto avaliado. Na ficha, a escala foi de 9 $\mathrm{cm}$ e os dados foram convertidos em números para análise estatística. As avaliações foram realizadas às 10 horas da manhã ou às 15 horas da tarde, buscando distanciá-las das principais refeições. As amostras forma servidas com "Dipas", salgadinho de milho comumente usado para acompanhar guacamole. Entre uma amostra e outra, os participantes da análise sensorial eram orientados a tomar água para evitar influência de sabor entre amostras provadas.

\section{Análise dos dados}

Os resultados da análise sensorial foram avaliados ao longo do período de armazenamento e por meio de uma análise de componentes principais (Mingoti, 2007), com o objetivo de buscar um número reduzido de combinações lineares das variáveis originais, capazes de explicar boa parte da variação contida nos dados. Baseado nos escores produzidos pela aplicação de tais componentes a cada uma das amostras, procedeu-se a uma análise de agrupamentos (Mingoti, 2007), visando a detectar grupos de amostras com avaliações semelhantes. As análises estatísticas foram executadas com o software R (R Development Core Team, 2007).

\begin{tabular}{|l|}
\hline Nome: \\
Teste Data_L___- \\
Aceitação__ \\
Textura \\
Cor \\
Sabor \\
Aparência__ \\
Comentários:
\end{tabular}

Figura 2 - Modelo ilustrativo da ficha de avaliação utilizada na análise sensorial do guacamole. 


\section{RESULTADOS E DISCUSSÕES}

A Figura 3 apresenta as notas de aceitação e sabor dos guacamoles refrigerados, submetidos ao congelamento lento e ao congelamento rápido. Sob refrigeração, o melhor tratamento foi aquele em que o guacamole permaneceu acondicionado em embalagem náilon+polietileno com vácuo, ficando nítida a inferioridade da embalagem de polietileno. Já nos congelamentos lento e rápido, a diferença entre tratamentos foi menor, sendo que o guacamole da embalagem de polietileno também obteve boa aceitabilidade. Observou-se diminuição da aceitabilidade ao longo do período de estocagem para todos os tratamentos, exceto no congelamento rápido, onde as notas permaneceram estáveis até os 90 dias. Já para o sabor, as amostras submetidas ao congelamento rápido com a embalagem de polietileno corresponderam ao tratamento que obteve as menores notas.

Para aparência e cor, observa-se, também, redução das notas dadas ao longo do período de estocagem. A embalagem com vácuo não apresentou superioridade em relação às demais (Figura 4).

A fim de extrair evidências relativas às associações existentes entre as variáveis em estudo, procedeu-
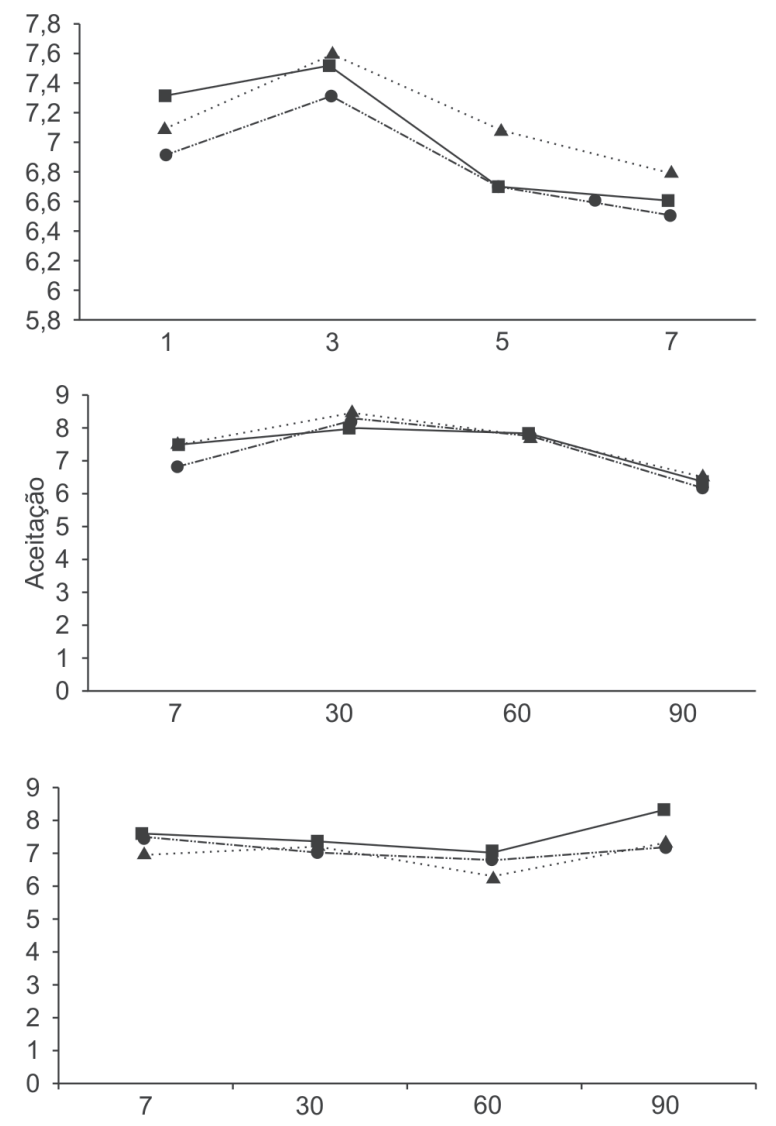

se a análise multivariada. A Figura 5 apresenta os gráficos de dispersão para cada par de variáveis sensoriais. As avaliações de textura e cor parecem apresentar relação linear positiva. À medida que aumenta o conceito atribuído à cor, o conceito atribuído à textura aumenta linearmente. Comportamento semelhante pode ser verificado para outros pares de variáveis, como cor e sabor ou aceitação e sabor, por exemplo.

Observa-se, na Tabela 1, correlação linear positiva moderada, entre algumas variáveis (r entre 0,40 e 0,62) e correlação forte entre outras ( $r$ entre 0,74 e 0,84 ). A maior correlação foi entre aceitação e sabor $(r=0,84)$, seguida da correlação entre cor e aparência $(\mathrm{r}=0,74)$. Daiuto et al. (2007), em análise de aceitação do guacamole elaborado apenas com limão, encontraram correlação entre aparência e sabor baixa $(0,35)$, melhorando para aceitação e sabor $(0,64)$. Para os autores, o fato foi indicativo de que a aparência do produto deveria ser melhorada.

Os dois primeiros componentes são capazes de conservar $84 \%$ da variação original dos dados, quantidade bastante expressiva, viabilizando o prosseguimento da análise com base nestas duas novas variáveis. A Tabela 2 apresenta a percentagem de variância dos dados originais, explicada por cada um dos cinco componentes obtidos.
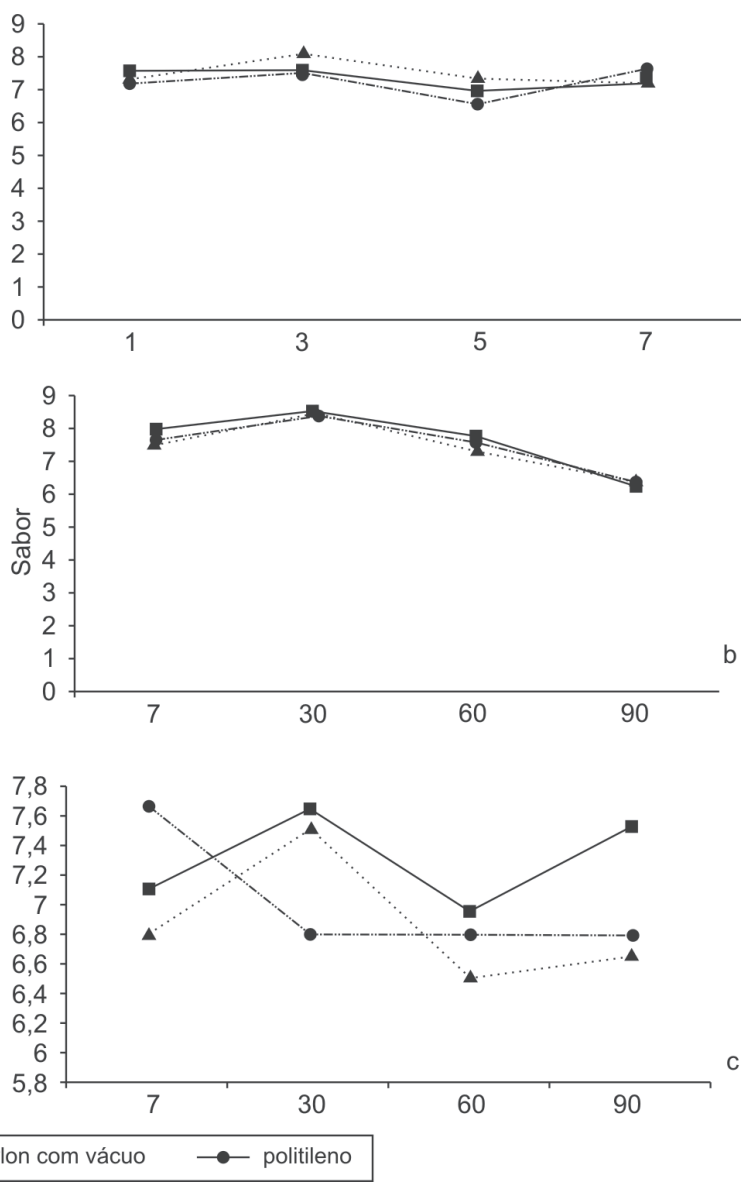

Figura 3 - Aceitação e sabor do guacamole refrigerado (a), submetido ao congelamento lento (b) e congelamento rápido (c) acondicionado em embalagens de polietileno, náilon+polietileno sem vácuo e náilon+polietileno com vácuo. 

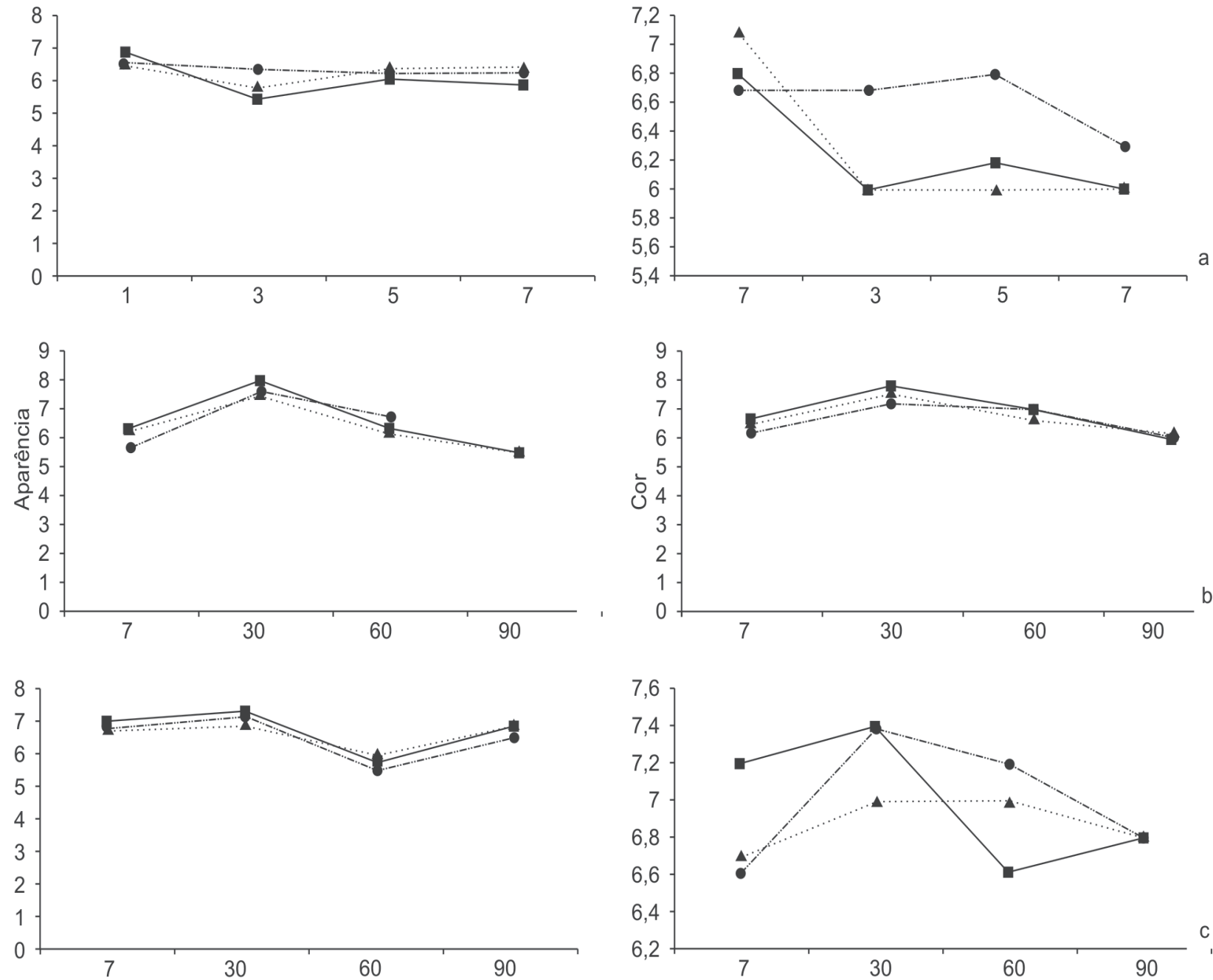

$\rightarrow$ nylon sem vácuo $\longrightarrow$ nylon com vácuo $\longrightarrow$ politileno

\section{dias de armazenamento}

Figura 4 - Aparência e cor do guacamole refrigerado (a), submetido ao congelamento lento (b) e congelamento rápido (c) acondicionado em embalagens de polietileno, náilon+polietileno sem vácuo e náilon+polietileno com vácuo.

Tabela 1 - Correlações lineares para os parâmetros de aceitação avaliados.

\begin{tabular}{lccccc}
\hline & Aceitação & Textura & Cor & Sabor & Aparência \\
\hline Aceitação & 1,00 & $0,66^{*}$ & $0,49^{*}$ & $0,84^{*}$ & $0,60^{*}$ \\
Textura & & 1,00 & $0,62^{*}$ & $0,60^{*}$ & $0,64^{*}$ \\
Cor & & 1,00 & $0,49^{*}$ & $0,74^{*}$ \\
Sabor & & & 1,00 & $0,57^{*}$ \\
Aparência & & & & 1,00 \\
\hline
\end{tabular}

$(\mathrm{P}<0,05)$

Tabela 2 - Percentagem da variância dos dados originais explicada por cada um dos cinco componentes obtidos

\begin{tabular}{lccccc}
\hline \multicolumn{5}{c}{ Componente } \\
\hline & 1 & 2 & 3 & 4 & 5 \\
Variância explicada (\%) & 70 & 14 & 7 & 5 & 3 \\
Variância explicada acumulada (\%) & 70 & 84 & 92 & 97 & 100 \\
Autovalores & 3,42 & 0,72 & 0,48 & 0,24 & 0,14 \\
\hline
\end{tabular}

Rev. Ceres, Viçosa, v. 58, n.2, p. 140-148, mar/abr, 2011 
As funções apresentadas na sequência expressam os dois componentes principais. A análise de seus coeficientes permite avaliar as correlações existentes entre as variáveis sensoriais.

Componente 1:

$C P 2=-0,46 *$ aceitação $+0,45 *$ textura $+0,42 *$ cor + $0,45 *$ sabor $+0,45 *$ aparência

O primeiro componente atribui ponderações positivas e de magnitudes semelhantes a cada uma das variáveis sensoriais consideradas, evidenciando correlações positivas entre as cinco variáveis sob estudo. Amostras de guacamole, apreciadas positivamente no conjunto de variáveis sensoriais, produzirão valores positivos com magnitudes grandes para este componente, enquanto amostras apreciadas negativamente fornecerão resultados negativos.

Componente 2:

$C P 2=-0,48 *$ aceitação $+0,06 *$ textura $+0,58 *$ cor + $0,51 *$ sabor $+0,39 *$ aparência

Observando as ponderações associadas a cada variável neste segundo componente, tem-se que a cor, a textura e aparência contribuem positivamente para ele. Amostras com valores menores para este componente têm aceitação bem avaliada.

A fim de identificar grupos com características semelhantes, para cada amostra de guacamole, foram calculados os respectivos escores para os dois componentes considerados. Com base nos escores obtidos, realizou-se uma análise de agrupamentos (Mingoti, 2007), com objetivo de detectar amostras de guacamole com avaliações semelhantes quanto às variáveis sensoriais (Figura 6). O método de agrupamento utilizado foi o de Ward (Mingoti, 2007) e optou-se, com base no dendrograma obtido, pela composição de nove clusters.

A Figura 7 apresenta os escores das amostras de guacamole em relação aos dois componentes principais. Amostras representadas por símbolos iguais pertencem a um mesmo grupo, dentre os nove fornecidos pela análise de agrupamentos.

O grupo 1, formado pelas amostras TCL30P, TCL30V, TCL30SV e T0, foi aquele que obteve as maiores notas para as variáveis sensoriais avaliadas, sendo de 7,9 a 8,4 para aceitação; 7,8 a 8,2 para textura; 7,2 a 8,2 para cor; 8,4 a 8,9 para sabor e 7,2 a 7,9 para aparência. O T0 corresponde à amostra avaliada no dia de produção. As amostras do congelamento lento, aos 30 dias, nas três embalagens, tiveram avaliação tão boa quanto esta amostra.

O grupo 2 foi formado pelas amostras TR1P, TR1V, TCR90P, TCR90V, TCR7V, TR1SV e TCR30V, sendo 4 amostras com congelamento rápido e 3 amostras refrigeradas. O grupo 3 foi formado pelas amostras TCR30P, TCR7SV e TCR30SV. O grupo 4 foi formado pelas amostras TR3P, TCL7V, TCL7SV, TCL60SV e TCL60V, com 4 amostras de congelamento lento e 1 refrigerada. $\mathrm{O}$ grupo 5 foi constituído pelas amostras TR7P, TCL60P, TCR7P e TCR90SV. Estes grupos apresentaram um comportamento intermediário, em relação aos outros grupos formados. Observou-se que constituem grupos em que a maioria das amos-

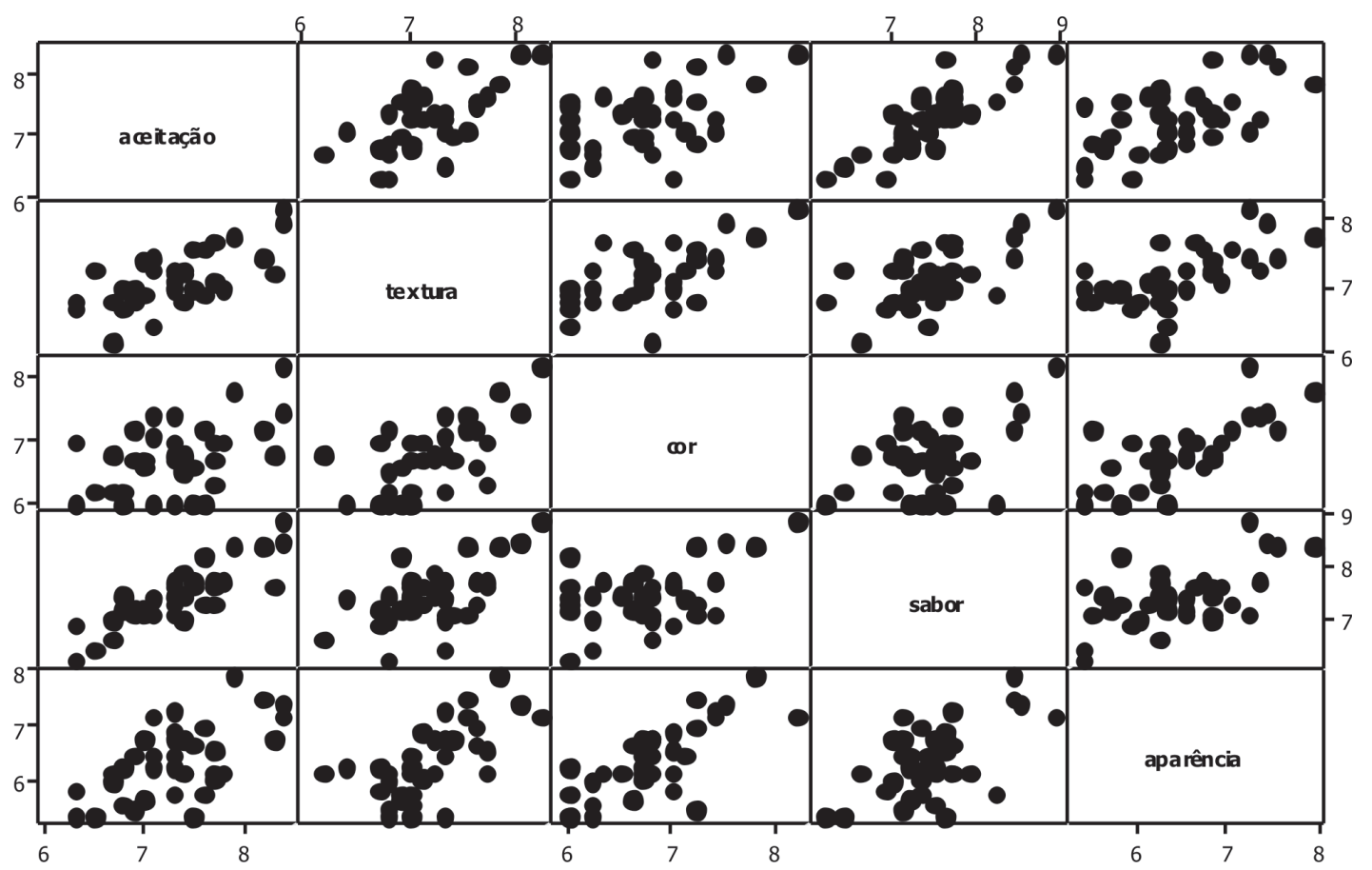

Figura 5 - Gráficos de dispersão para as variáveis sensoriais. 
tras são as refrigeradas ou aquelas com tempo de armazenamento superior a 60 dias.

Os grupos 6, 7, 8 e 9 foram formados, respectivamente, pelas amostras TR5P, TCR60P e TCR60V; TCL90SV e TCL90V; TR3SV, TR7SV e TR3V; TR5SV, TR7V, TR5V, TCL7P e TCR60SV e apresentaram as menores notas para aparência $(5,4-6,2)$, aceitação $(6,3-7,5)$ e sabor (menor nota foi 5,4 )

Pôde-se observar que amostras acondicionadas em embalagens com barreira contra gases, com ou sem vácuo, não permaneceram todas num grupo de melhores notas, e aquelas acondicionadas em embalagem de polietileno nem sempre estiveram nos grupos de menores notas para as variáveis sensoriais. Isso indica um efeito positivo da adição do $\alpha$-tocoferol e do ácido ascórbico para amostras da embalagem de polietileno, pois, algumas vezes, foram tão bem apreciadas quanto as das outras embalagens. O tempo de armazenamento, seja nas amostras refrigeradas, seja nas congeladas, foi o fator de maior influência na qualidade do guacamole e na separação dos grupos.

Para Daiuto et al (2007), que realizaram avaliação sensorial de guacamole sem aditivos, usando apenas limão na formulação, a embalagem de polietileno mostrou as menores notas para os parâmetros sensoriais avaliados.

Amostras refrigeradas, a partir do $5^{\circ}$ dia ,e congeladas, após os 60 dias, de modo geral ficaram em grupos de notas, de intermediárias a inferiores ao grupo 5, cujas notas para os parâmetros avaliados foram as mais elevadas. Uma das alterações sensoriais no produto armazenado é de

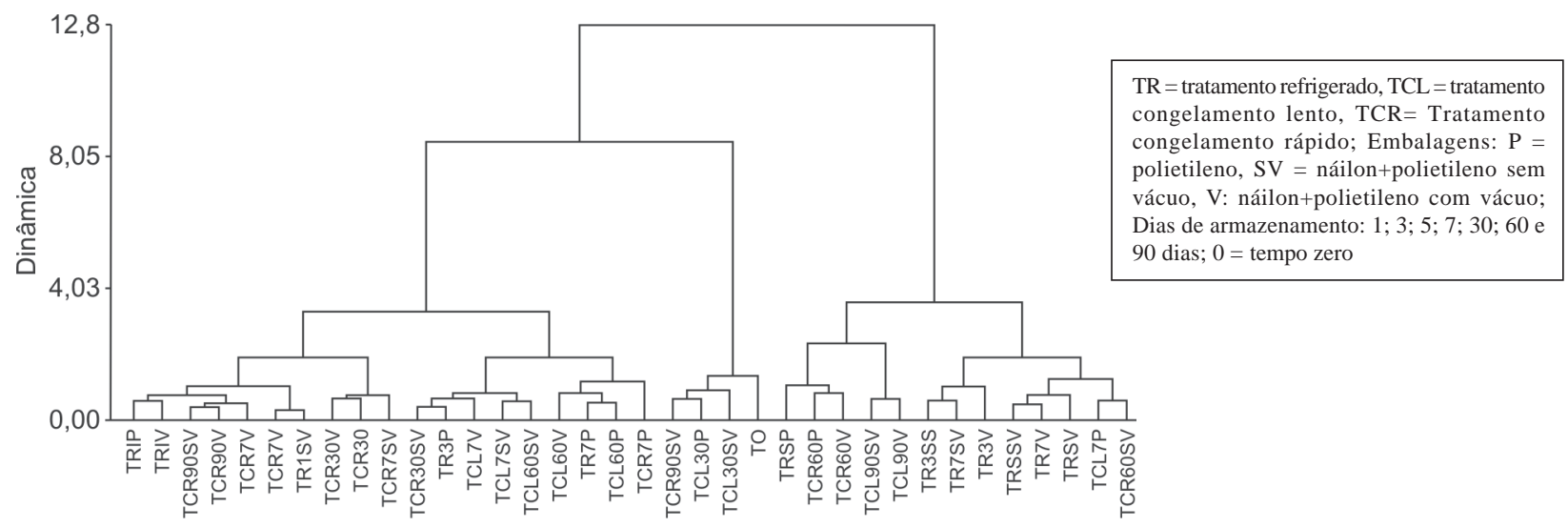

Figura 6 - Dendrograma da análise de agrupamentos, baseada nos escores calculados sob os dois componentes principais, para cada amostra de guacamole.

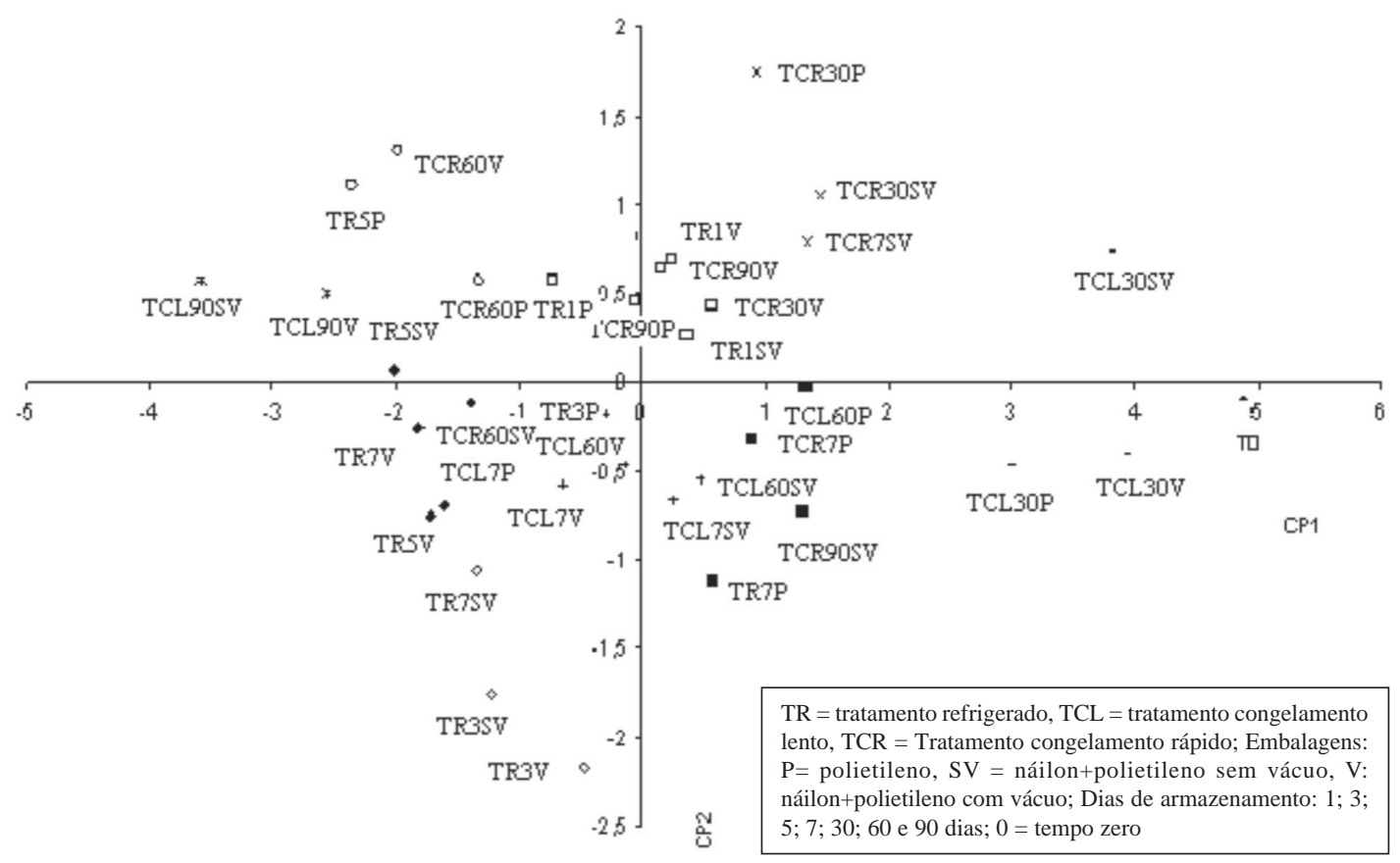

Figura 7 - Escores (para cada amostra de guacamole) e coeficientes (para cada variável) associados aos dois componentes principais (84\% da variação total). 
seu sabor. Pode ter ocorrido aumento da acidez da amostra, por quebra de lipídios e liberação de ácidos graxos e, neste caso, a adição de $\alpha$-tocoferol pode não ter sido efetiva para evitar a rancidez.

Flores et al. (2008) realizaram análise sensorial, utilizando também uma escala hedônica de 9 pontos, em guacamole adicionado de antioxidantes, ácido ascórbico e sulfito de sódio, para inibir o escurecimento. As notas para os parâmetros de aceitação foram baixas e diminuíram, da média de 6,45 , do dia de elaboração do produto, para 3,65, aos 10 dias após o armazenamento. A adição de ácido ascórbico e sulfito de sódio permitiu a conservação do produto por 10 dias $\left(5^{\circ} \mathrm{C} \pm 1\right)$, mas o sabor característico do produto manteve-se apenas por 7 dias.

Ramthal et al. (2007) elaboraram guacamole, sem aditivos químicos, conservado sob refrigeração $\left(5^{\circ} \mathrm{C}\right)$ e em embalagem pet (polyethylene terephthalate). As amostras foram avaliadas quanto a aparência, cor, aroma, consistência, textura e aceitabilidade geral. A avaliação sensorial revelou que a média de período de armazenamento adequada, nesta temperatura, era de 6 dias. Após este período, a textura e aroma eram deteriorados, principalmente por causa da produção de off flavor, resultante da hidrólise de ácidos graxos e da oxidação de compostos fenólicos.

Elez-Martinez et al. (2005) concluíram que a adição de 100 ppm de $\alpha$-tocoferol, ou 200 ppm de ácido ascórbico, pode estabilizar o produto, do ponto de vista de rancidez, por até 24 semanas, sob armazenamento refrigerado. Apesar de o $\alpha$-tocoferol ser o mais efetivo antioxidante, o ácido ascórbico tem a vantagem de ter efeito benéfico também na prevenção do escurecimento da polpa. Estes autores utilizaram embalagem de polietileno, que foram seladas, com e sem vácuo. O resultado positivo destes autores pode ser devido ao fato de o produto elaborado ser uma pasta e a homogeinização dos ingredientes ter sido mais efetiva, em relação à presente pesquisa, pois, no guacamole, a polpa é desintegrada apenas em pedaços.

A adição do $\alpha$-tocoferol parece não ter sido efetiva para manutenção do sabor do produto, após 30 dias de armazenamento. $\mathrm{O}$ tocoferol é encontrado naturalmente em óleos vegetais e tem efeito antioxidante, interagindo com radicais livres, oriundos da oxidação de lipídios (Araújo, 2006). No experimento, também foi observada dificuldade no uso do $\alpha$-tocoferol. Trata-se de um aditivo caro, o que inviabilizaria a comercialização do guacamole, e é de difícil dissolução/homogeinização na formulação. Essa dificuldade pode explicar o fato de algumas amostras apresentarem boa avaliação, em embalagem de polietileno, e, às vezes, pior avaliação em embalagem com barreira contra gases e presença de vácuo.

\section{CONCLUSÕES}

Com a análise multivariada dos dados, observou-se que amostras acondicionadas em embalagens com barreira contra gases, com ou sem vácuo, não permaneceram todas num grupo de melhores notas. O tempo de armazenamento, seja nas amostras refrigeradas, seja nas congeladas, foi o fator de maior influência na qualidade do guacamole e na separação dos grupos. Observou-se a influência do sabor na aceitação do guacamole, uma vez que houve uma correlação positiva entre as duas variáveis. O produto, submetido ao congelamento lento, conservado até 30 dias em qualquer embalagem, apresentou médias de aceitabilidade, comparadas com a da avaliação do primeiro dia em que foi elaborado o produto. Apesar de notas inferiores de aceitação, a adição de ácido ascórbico e $\alpha$-tocoferol permite a conservação do produto, mesmo sob refrigeração e em embalagens de polietileno.

\section{AGRADECIMENTOS}

À empresa Jaguacy (Bauru-SP), pelo apoio e participação nas pesquisas; à CAPES e à Fundação de Apoio a Pesquisa no Estado de São Paulo.

\section{REFERÊNCIAS}

Araújo JMA (2006) Química de alimentos: teoria e prática. 3.ed. Viçosa, UFV. 478p.

Bates RP (1968) The retardation of enzymatic browning in avocado puree and guacamole. Proceeding Florida State Horticultural Society, 81:30-235.

Ben-Et G, Dolev A \& Tatarsk D (1973) Compounds contributing to heat-induced bitter off-flavor in avocado. Journal of Food Science, 38:546-5547.

Chaves JBP \& Sproesser RL (1999) Práticas de laboratório de análise sensorial de alimentos e bebidas. Viçosa, UFV. p.45-46. (Cadernos Didáticos, 66)

Daiuto ER, Vieites RL, Taconelli C, Gonçalvez AdeF, Piveta PR \& Simon JW (2007). Avaliação sensorial do guacamole conservado pelo frio. Revista de Alimentos e Nutrição, 18:405-412.

Elez-Martinez P, Soliva-Fortuny RC, Gorintein S \& Martín-Belloso O (2005). Natural antioxidants preserve the lipid oxidative stability of minimally processed avocado purê. Journal of Food Science, 70:325-329.

Flores JM, Orozco ML, Soto EM \& Gonzalez MdeLA (2008) Tecnología antiobscurecimento en una formulation de pasta de aguacate (guacamole). Disponível em: <www.respyn.uanl.mx/ especiales/2006/ee-14-2006/documentos/Art34.pdf->. Acessado em: 12 de setembro 2009.

Gómez-López VM (2002) Some biochemical proprierties of polyphenol oxidase from two varieties of avocado. Food Chemistry, 77:163-169.

Guzmán GR, Dorantes AL, Hernandez UH, Ortiz A \& Mora ER (2002) Effect of zinc and copper chloride on the color of avocado puree heated with microwaves. Innovative Food Science and Emerging Technologies, 3:47-53. 
Kader AA (1992) Postharvest technology of horticultural crops. Okland, University of California. 292p.

Kluge RA, Jacomino AP, Ojeda RM \& Brackamann A (2002) Inibição do amadurecimento de abacate com 1-metilciclopropeno. Pesquisa Agropecuária Brasileira, 37:895-901.

Palou E, Hérnandez-Salgado C, López- Malo A, Barbosa-Canóvas GV, Swanson BG \& Welt-Chanes J (2000) High pressureprocessed guacamole. Innovative Food Science and Emerging Technologies, 1:69-75.

López-Malo A, Palou E, Barbosa G, Welti J \& Swanson B (1998) Polyphenoloxidase activity and color changes during of high hydrostatic pressure treated avocado puree. Food Research International, 31:549-56.

Luíz RC, Hirata TAM \& Clemente E (2007) Cinética de inativação da polifenoloxidase e peroxisase de abacate (Persea americana Mill.). Ciência e Agrotecnologia, 31:1766-1773.

Mingoti (2007). Análise de dados através de métodos de estatística multivariada: uma abordagem aplicada. Belo Horizonte, UFMG. 295p

R Development Core Team (2007) R: A language and environment for statistical computing. R Foundation for Statistical Computing, Vienna, Austria. ISBN 3-900051-07-0. Disponível em: L <http:/ /www.R-project.org >.Acessado em: 15 de setembro 2008.
Ramthal GA, Akingbala JO \& Baccus-Taylor GS (2007) Laboratory preparation and evaluation of Pollock variety avocado (Persea americana Mill). Journal of the Science of Food and Agriculture, 87:2068-2074.

Reginato-D'arce MAB (2006) Deterioração de lipídios-ranço. In; Oetterer M, Reginato-D'arce Mab \& Spoto MHF (Eds.) Fundamentos de Ciência e Tecnologia de Alimentos. Barueuri, Manole. p.243-299.

Soliva RC, Elez-Martínez P, Sebastían-Calderó M \& Martín-Belloso O (2001) Evaluation of browning effect on avocado purée preserved by combined methods. Innovative Food Science Emerging Technology, 1:261-268.

Soliva-Fortuny RC, Elez-Martínez P, Sebastían-Calderó, M \& Martín-Belloso O (2004) Effect of combined methods of preservation on the naturally occurring microflora of avocado purée. Food Control, 15:11-17.

Schwenke DC (1998) Antioxidants and atherogenesis. The Journal of Nutritional Biochemistry, 9:424-445.

Son Moon K \& Lee C (2000) Kinetic study of oxalic acid inhibition on enzymatic browning. Journal of Agricultural and Food Chemistry, 48:2071-2074.

Stephens TS, Lime BJ \& Griffiths FP (1958) The effect of thickening agents in reducing the watery separation of frozen and thawed guacamole products. Proceedings of the Rio Grande Valley Horticultural Society, 12:81-87. 